\title{
Vibration control strategy for large-scale structures with incomplete multi-actuator system and neighbouring state information
}

\author{
Francisco Palacios-Quiñonero ${ }^{1,3}$ Josep Rubió-Massegú ${ }^{1,4}$ \\ Josep M. Rossel1 ${ }^{1,5}$ and Hamid Reza Karimi ${ }^{2,6}$
}

\begin{abstract}
The synthesis of optimal controllers for vibrational protection of large-scale structures with multiple actuation devices and partial state information is a challenging problem. In this paper, we present a design strategy that allows computing this kind of controllers by using standard linear matrix inequality optimization tools. To illustrate the main elements of the new approach, a five-story structure equipped with two interstory actuation devices and subjected to a seismic disturbance is considered. For this control setup, three different controllers are designed: an ideal state-feedback $H_{\infty}$ controller with full access to the complete state information and two static output-feedback $H_{\infty}$ controllers with restricted neighbouring state information. To assess the performance of the proposed controllers, the corresponding frequency responses are investigated and a proper set of numerical simulations are conducted, using the full scale North-South El Centro 1940 seismic record as ground acceleration input. The obtained results indicate that, despite the severe information constraints, the proposed static output-feedback controllers attain a level of seismic protection that is very similar to that achieved by the ideal state-feedback controller with complete state information.
\end{abstract}

Keywords: structural vibration control; large-scale control; partial state information; static output-feedback control; LMI optimization

\section{Introduction}

Nowadays, advanced strategies for vibration control of large mechanical structures can involve complex actuation systems, consisting in several active or semiactive devices that work coordinately to mitigate the vibrational response of the overall structure. Good examples of this approach are provided by feedback control systems for seismic protection of tall buildings [1-3]. In the most traditional designs, the large control forces required to mitigate the structural vibration response are produced by means of a single actuation device, placed either at ground level or at the top of the structure [4-7]. In contrast, a system of several medium-size actuation devices is frequently considered in more advanced designs [8-10]. In this context, interstory actuation devices are of particular interest. These devices can be placed between any pair of consecutive stories and, in addition to exerting the corresponding structural control forces, they can also include the following elements: (i) a sensing unit that facilitates gathering information of the local interstory drifts and interstory velocities, (ii) a communication unit for information exchange with neighbouring devices and (iii) a local control unit that allows computing the corresponding control action using preferably local and neighbouring state information [11,12].

In fully instrumented designs, interstory devices are implemented at all levels of the structure. This control setup, schematically depicted in Figure 1(a), includes a complete actuation system and, also, a complete system of sensors, which can give access to the full state information. Using advanced control design methodologies based on linear matrix inequality (LMI) formulations, state-feedback controllers can be effectively obtained for multi-actuator control architectures with complete state information [13]. Moreover, the LMI solvers allow defining sparsity patterns on the control gain matrices

\footnotetext{
${ }^{1}$ Department of Applied Mathematics III, Universitat Politècnica de Catalunya (UPC), Av. Bases de Manresa 61-73, 08242 Manresa, Barcelona, Spain.

${ }^{2}$ Department of Engineering, Faculty of Engineering and Science, University of Agder (UiA), 4898 Grimstad, Norway.

${ }^{3}$ Email: francisco.palacios@upc.edu

${ }^{4}$ Email: josep.rubio@upc.edu

${ }^{5}$ Email: josep.maria.rossell@upc.edu

${ }^{6}$ Corresponding author, email: hamid.r.karimi@uia.no
} 
(a)

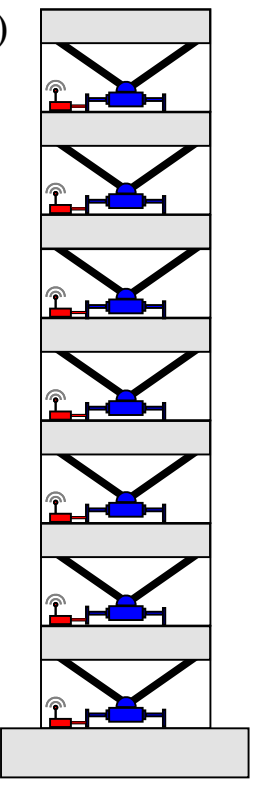

(b)

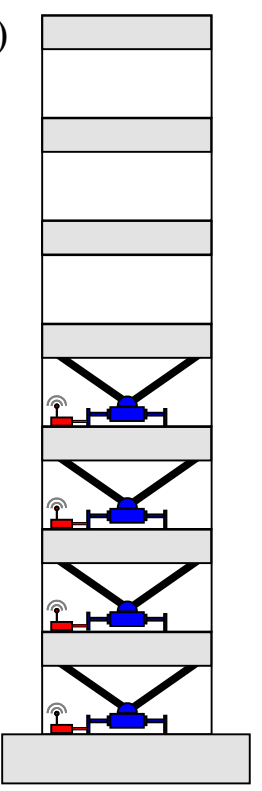

Figure 1: (a) Fully instrumented structure with complete systems of actuators and sensors. (b) Partially instrumented structure with incomplete systems of actuators and sensors

to obtain decentralized or semidecentralized controllers that, for a given actuation device, make it possible to compute the corresponding control actions using neighbouring state information provided by the local sensor or by sensors located in adjacent stories [14-16].

In partially instrumented designs, as the one displayed in Figure 1(b), an incomplete system of interstory devices is implemented, which allows exerting control forces at selected levels of the structure and acquiring partial state information. Even for a moderate number of stories, a rich variety of control setups can be obtained in this case, and designs with different degrees of complexity can be considered for a given control problem. The potential benefits provided by this broader approach, however, are seriously compromised by the challenging theoretical and computational issues associated to the design of optimal controllers with partial state information [17-20].

The objective of the present paper is to provide an advanced computational strategy to deal with the complex problem of designing optimal static feedback controllers for vibrational protection of partially instrumented large-scale structures, which are equipped with incomplete multi-actuator and multi-sensor systems and are naturally subject to severe restrictions on the available state information. The proposed solution is based on recent advances in static output-feedback control [21, 22], which facilitate designing controllers of the form

$$
\mathbf{u}(t)=\mathbf{K} \mathbf{y}(t),
$$

where the vector $\mathbf{u}(t)$ contains the control actions corresponding to the incomplete actuation system, $\mathbf{K}$ is a constant matrix, and $\mathbf{y}(t)$ is a vector that contains the partial state information provided by the incomplete system of sensors. The design methodology comprises two successive steps. First, a satisfactory state-feedback controller

$$
\mathbf{u}(t)=\widetilde{\mathbf{G}}_{s} \mathbf{x}(t)
$$

is obtained, where $\mathbf{x}(t)$ is the state vector and $\widetilde{\mathbf{G}}_{s}$ is a constant matrix. As previously mentioned, this ideal controller with complete state information can be effectively computed by solving a standard LMI optimization problem $\mathscr{P}_{s}$. Next, by introducing a suitable transformation of the LMI variables in $\mathscr{P}_{s}$, a second LMI optimization problem $\mathscr{P}_{o}$ is derived, which allows obtaining an almost-optimal static output-feedback controller with partial state information.

From a formal point of view, controllers with neighbouring state information can be considered as a particular case of state-feedback controllers with information constraints and, in principle, they could be obtained by setting an appropriate sparsity pattern on the state-feedback control gain matrix. However, even in the simplest cases, these constrained LMI 
optimization problems are reported to be infeasible by the Matlab LMI solver. In contrast, using the proposed computational strategy, a wide variety of controller designs with different neighbouring control information structures can be obtained with no feasibility issues.

For brevity and clarity, a five-story structure model with interstory devices implemented at first and second levels has been chosen to introduce the main ideas (see Figure 2). For this particular control setup, two different $H_{\infty}$ controllers with partial state information are designed: (i) a static output-feedback controller that uses the interstory drifts and interstory velocities of the first and second levels as measured outputs, and (ii) a static velocity-feedback controller that only uses the interstory velocities of the first and second levels as feedback information. Moreover, an ideal state-feedback $H_{\infty}$ controller with full access to the state information is computed in the first step of the design procedure.

To assess the effectiveness of the proposed output-feedback controllers with neighbouring state information, the corresponding frequency responses are investigated and a proper set of numerical simulations are carried out using the full-scale Noth-South El Centro 1940 seismic record as ground acceleration input. In all the cases, the behaviour of the ideal statefeedback $H_{\infty}$ controller is taken as a reference. When convenient, the numerical details of the different controller designs are complemented with a minimal theoretical background to provide a more self-contained presentation.

Although the discussion has been focused on the problem of building seismic protection, it is worth to be highlighted that the proposed approach can also produce positive results in other physical applications involving large structures subjected to environmental excitations such as wind gusts or marine waves. Examples of clear practical interest include tall antennas and communication towers, inland and offshore wind turbines for energy generation and large marine platforms [23-25]. Moreover, control strategies with neighbouring state information require shorter communication ranges and, consequently, they are particularly suitable for wireless implementations of the communication system $[11,12,14]$.

The paper is organized as follows: In Section 2, a mathematical model of the partially instrumented five-story structure is provided. In Section 3, the LMI optimization problem $\mathscr{P}_{s}$ is presented and a suitable state-feedback $H_{\infty}$ controller with full state information is designed. In Section 4, the general form of the LMI optimization problem $\mathscr{P}_{o}$ is briefly discussed and the first output-feedback controller is computed. In Section 5, a controller with neighbouring velocity-feedback information is synthesized. Finally, in Section 6, some conclusions and future research directions are presented.

\section{Structure model}

Let us consider the five-story structure schematically depicted in Figure 2, where we assume that an actuation device $a_{i}$ has been implemented between the consecutive stories $s_{i-1}$ and $s_{i}$ for $i=1,2$. The actuation device $a_{i}$ exerts a control action $u_{i}(t)$, which produces a pair of opposite structural forces as indicated in the figure. By defining the vector of displacements

$$
\mathbf{q}(t)=\left[q_{1}(t), q_{2}(t), q_{3}(t), q_{4}(t), q_{5}(t)\right]^{T}
$$

where $q_{i}(t)$ is the lateral displacement of the story $s_{i}$ with respect to the ground level $s_{0}$, the lateral motion of the structure can be described by the differential equation

$$
\mathbf{M} \ddot{\mathbf{q}}(t)+\mathbf{C}_{d} \dot{\mathbf{q}}(t)+\mathbf{K}_{s} \mathbf{q}(t)=\mathbf{T}_{u} \mathbf{u}(t)+\mathbf{T}_{w} w(t),
$$

where the vector of control actions has the form

$$
\mathbf{u}(t)=\left[u_{1}(t), u_{2}(t)\right]^{T}
$$

and $w(t)$ denotes the seismic ground acceleration. $\mathbf{M}, \mathbf{C}_{d}$ and $\mathbf{K}_{s}$ are the mass, damping and stiffness matrices, respectively, $\mathbf{T}_{u}$ is the control location matrix and $\mathbf{T}_{w}$ is the excitation location matrix. Let us now consider the interstory drifts

$$
\left\{\begin{array}{l}
r_{1}(t)=q_{1}(t) \\
r_{i}(t)=q_{i}(t)-q_{i-1}(t) \text { for } i=2, \ldots, 5,
\end{array}\right.
$$

the interstory velocities

$$
v_{i}(t)=\dot{r}_{i}(t), \quad i=1, \ldots, 5
$$




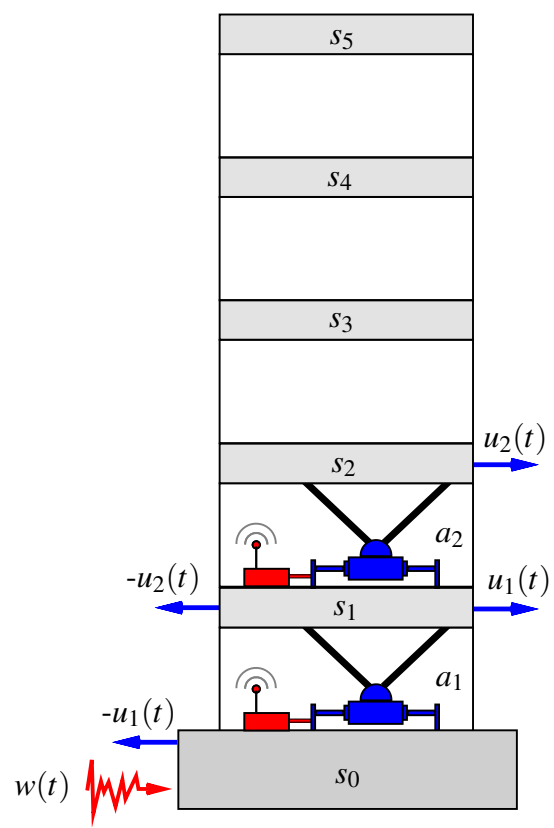

Figure 2: Partially instrumented five-story structure with interstory actuators and sensors implemented at first and second levels

and the state vector

$$
\mathbf{x}(t)=\left[r_{1}(t), v_{1}(t), r_{2}(t), v_{2}(t), \ldots, r_{5}(t), v_{5}(t)\right]^{T}
$$

that groups together the interstory drifts and interstory velocities of a same level. Using the state vector $\mathbf{x}(t)$, we can derive a first-order state-space model

$$
\dot{\mathbf{x}}(t)=\mathbf{A} \mathbf{x}(t)+\mathbf{B}_{u} \mathbf{u}(t)+\mathbf{B}_{w} w(t),
$$

with

$$
\begin{gathered}
\mathbf{A}=\mathbf{P} \widehat{\mathbf{A}} \mathbf{P}^{-1}, \quad \mathbf{B}_{u}=\mathbf{P} \widehat{\mathbf{B}}_{u}, \quad \mathbf{B}_{w}=\mathbf{P} \widehat{\mathbf{B}}_{w}, \\
\widehat{\mathbf{A}}=\left[\begin{array}{cc}
{[\mathbf{0}]_{5 \times 5}} & \mathbf{I}_{5} \\
-\mathbf{M}^{-1} \mathbf{K}_{s} & -\mathbf{M}^{-1} \mathbf{C}_{d}
\end{array}\right], \\
\widehat{\mathbf{B}}_{u}=\left[\begin{array}{c}
{[\mathbf{0}]_{5 \times 2}} \\
\mathbf{M}^{-1} \mathbf{T}_{u}
\end{array}\right], \quad \widehat{\mathbf{B}}_{w}=\left[\begin{array}{r}
{[\mathbf{0}]_{5 \times 1}} \\
-[\mathbf{1}]_{5 \times 1}
\end{array}\right],
\end{gathered}
$$

where $[\mathbf{0}]_{n \times m}$ represents a zero-matrix of the indicated dimensions, $\mathbf{I}_{n}$ is the identity matrix of order $n,[\mathbf{1}]_{n \times 1}$ denotes a vector of dimension $n$ with all its entries equal to 1 and $\mathbf{P}$ is the change of basis matrix

$$
\mathbf{P}=\left[\begin{array}{rrrrrrrrrr}
1 & 0 & 0 & 0 & 0 & 0 & 0 & 0 & 0 & 0 \\
0 & 0 & 0 & 0 & 0 & 1 & 0 & 0 & 0 & 0 \\
-1 & 1 & 0 & 0 & 0 & 0 & 0 & 0 & 0 & 0 \\
0 & 0 & 0 & 0 & 0 & -1 & 1 & 0 & 0 & 0 \\
0 & -1 & 1 & 0 & 0 & 0 & 0 & 0 & 0 & 0 \\
0 & 0 & 0 & 0 & 0 & 0 & -1 & 1 & 0 & 0 \\
0 & 0 & -1 & 1 & 0 & 0 & 0 & 0 & 0 & 0 \\
0 & 0 & 0 & 0 & 0 & 0 & 0 & -1 & 1 & 0 \\
0 & 0 & 0 & -1 & 1 & 0 & 0 & 0 & 0 & 0 \\
0 & 0 & 0 & 0 & 0 & 0 & 0 & 0 & -1 & 1
\end{array}\right]
$$

corresponding to the state transformation

$$
\mathbf{x}(t)=\mathbf{P}\left[\begin{array}{c}
\mathbf{q}(t) \\
\dot{\mathbf{q}}(t)
\end{array}\right]
$$


The mass and stiffness matrices have the following form:

$$
\begin{gathered}
\mathbf{M}=\left[\begin{array}{ccccc}
m_{1} & 0 & 0 & 0 & 0 \\
0 & m_{2} & 0 & 0 & 0 \\
0 & 0 & m_{3} & 0 & 0 \\
0 & 0 & 0 & m_{4} & 0 \\
0 & 0 & 0 & 0 & m_{5}
\end{array}\right], \\
\mathbf{K}_{s}=\left[\begin{array}{ccccc}
k_{1}+k_{2} & -k_{2} & 0 & 0 & 0 \\
-k_{2} & k_{2}+k_{3} & -k_{3} & 0 & 0 \\
0 & -k_{3} & k_{3}+k_{4} & -k_{4} & 0 \\
0 & 0 & -k_{4} & k_{4}+k_{5} & -k_{5} \\
0 & 0 & 0 & -k_{5} & k_{5}
\end{array}\right],
\end{gathered}
$$

where $m_{i}, k_{i}, i=1, \ldots, 5$ denote the mass and stiffness coefficients of the $i$-th story, respectively. For the building model used in this paper, the mass and stiffness values presented in Table 1 are considered [26]. To compute the damping matrix, a Rayleigh damping matrix with a $2 \%$ damping ratio on the first and fifth modes has been used [27], resulting:

$$
\mathbf{C}_{d}=10^{3} \times\left[\begin{array}{ccccc}
260.2 & -92.4 & 0 & 0 & 0 \\
-92.4 & 219.6 & -81.0 & 0 & 0 \\
0 & -81.0 & 199.5 & -72.8 & 0 \\
0 & 0 & -72.8 & 186.7 & -68.7 \\
0 & 0 & 0 & -68.7 & 127.4
\end{array}\right]
$$

Finally, the control location matrix and the excitation location matrix are

$$
\mathbf{T}_{u}=\left[\begin{array}{rr}
1 & -1 \\
0 & 1 \\
0 & 0 \\
0 & 0 \\
0 & 0
\end{array}\right], \quad \mathbf{T}_{w}=-\mathbf{M}\left[\begin{array}{l}
1 \\
1 \\
1 \\
1 \\
1
\end{array}\right]
$$

For the parameter values in Table 1 and the matrices in (17) and (18), the following system matrices result:

$$
\begin{aligned}
& \mathbf{A}=10^{3} \times\left[\begin{array}{ccccccccccc}
0 & 0.0010 & 0 & 0 & 0 & 0 & 0 & 0 & 0 & 0 \\
-0.6831 & -0.0008 & 0.5251 & 0.0004 & 0 & 0 & 0 & 0 & 0 & 0 \\
0 & 0 & 0 & 0.0010 & 0 & 0 & 0 & 0 & 0 & 0 \\
0.6831 & 0.0006 & -1.0652 & -0.0011 & 0.4732 & 0.0004 & 0 & 0 & 0 & 0 \\
0 & 0 & 0 & 0 & 0 & 0.0010 & 0 & 0 & 0 & 0 \\
0 & 0 & 0.5402 & 0.0004 & -0.9515 & -0.0010 & 0.4300 & 0.0004 & 0 & 0 \\
0 & 0 & 0 & 0 & 0 & 0 & 0 & 0.0010 & 0 & 0 \\
0 & 0 & 0 & 0 & 0.4783 & 0.0004 & -0.8645 & -0.0009 & 0.4102 & 0.0003 \\
0 & 0 & 0 & 0 & 0 & 0 & 0 & 0 & 0 & 0.0010 \\
0 & 0 & 0 & 0 & 0 & 0 & 0.4346 & 0.0004 & -0.7258 & -0.0008
\end{array}\right], \\
& \mathbf{B}_{u}=10^{-5} \times\left[\begin{array}{cc}
0 & 0 \\
0.4647 & -0.4647 \\
0 & 0 \\
-0.4647 & 0.9427 \\
0 & 0 \\
0 & -0.4780 \\
0 & 0 \\
0 & 0 \\
0 & 0 \\
0 & 0
\end{array}\right], \quad \mathbf{B}_{w}=\left[\begin{array}{c}
0 \\
-1 \\
0 \\
0 \\
0 \\
0 \\
0 \\
0 \\
0 \\
0
\end{array}\right] .
\end{aligned}
$$


Table 1: Mass and stiffness coefficient values

\begin{tabular}{rccccc}
\hline & \multicolumn{5}{c}{ story } \\
\cline { 2 - 6 } & 1 & 2 & 3 & 4 & 5 \\
\hline Mass $\left(\times 10^{5} \mathrm{Kg}\right)$ & 2.152 & 2.092 & 2.070 & 2.048 & 2.661 \\
Stiffness $\left(\times 10^{8} \mathrm{~N} / \mathrm{m}\right)$ & 1.470 & 1.130 & 0.990 & 0.890 & 0.840 \\
\hline
\end{tabular}

\section{State-feedback $H_{\infty}$ controller}

In this section, we compute a state-feedback $H_{\infty}$ controller for the proposed five-story structure model. This ideal controller has a complete access to the state information and will be taken as a reference in the performance assessment of the static output-feedback controllers with partial state information designed in Sections 4 and 5. Moreover, the LMI optimization problem $\mathscr{P}_{s}$ associated to the state-feedback design provides a matrix $\widetilde{\mathbf{X}}_{s}$ that facilitates a suitable definition of the LMI variable transformations required to obtain the static output-feedback controllers. All the controllers presented in this paper are computed with the LMI optimization tools provided by the Matlab Robust Optimization Toolbox [28].

Assuming that we are interested in minimizing the building vibrational response and the control efforts, we introduce the vector of controlled outputs

$$
\mathbf{z}(t)=\mathbf{C}_{z} \mathbf{x}(t)+\mathbf{D}_{z} \mathbf{u}(t)
$$

where

$$
\mathbf{C}_{z}=\left[\begin{array}{c}
\mathbf{I}_{10} \\
{[\mathbf{0}]_{2 \times 10}}
\end{array}\right], \quad \mathbf{D}_{z}=\alpha\left[\begin{array}{c}
{[\mathbf{0}]_{10 \times 2}} \\
\mathbf{I}_{2}
\end{array}\right],
$$

and $\alpha>0$ is a suitable coefficient that trades-off the conflicting design objectives. Here, we aim at computing an optimal state-feedback controller

$$
\mathbf{u}(t)=\widetilde{\mathbf{G}}_{s} \mathbf{x}(t)
$$

for the state-space system given in (9), using the controlled output $\mathbf{z}(t)$ to define the performance criteria. For a given state-feedback controller

$$
\mathbf{u}(t)=\mathbf{G} \mathbf{x}(t)
$$

we obtain the closed-loop system

$$
\left\{\begin{array}{l}
\dot{\mathbf{x}}(t)=\mathbf{A}_{G} \mathbf{x}(t)+\mathbf{B}_{w} w(t) \\
\mathbf{z}_{G}(t)=\mathbf{C}_{G} \mathbf{x}(t)
\end{array}\right.
$$

where

$$
\mathbf{A}_{G}=\mathbf{A}+\mathbf{B}_{u} \mathbf{G}, \quad \mathbf{C}_{G}=\mathbf{C}_{z}+\mathbf{D}_{z} \mathbf{G} .
$$

The $H_{\infty}$-norm of the state-feedback controller defined by the state gain matrix $\mathbf{G}$ is the largest energy gain from the disturbance input to the controlled output

$$
\gamma_{G}=\sup _{\|w\|_{2} \neq 0} \frac{\left\|\mathbf{z}_{G}\right\|_{2}}{\|w\|_{2}}
$$

where $\|\cdot\|_{2}$ is the usual continuous 2-norm

$$
\|\mathbf{g}\|_{2}=\left[\int_{0}^{\infty}\{\mathbf{g}(t)\}^{T} \mathbf{g}(t) d t\right]^{1 / 2} .
$$

Using the closed-loop transfer function from the disturbance input $w(t)$ to the controlled output $\mathbf{z}_{G}(t)$

$$
\mathbf{T}_{G}(s)=\mathbf{C}_{G}\left(s \mathbf{I}-\mathbf{A}_{G}\right)^{-1} \mathbf{B}_{w},
$$




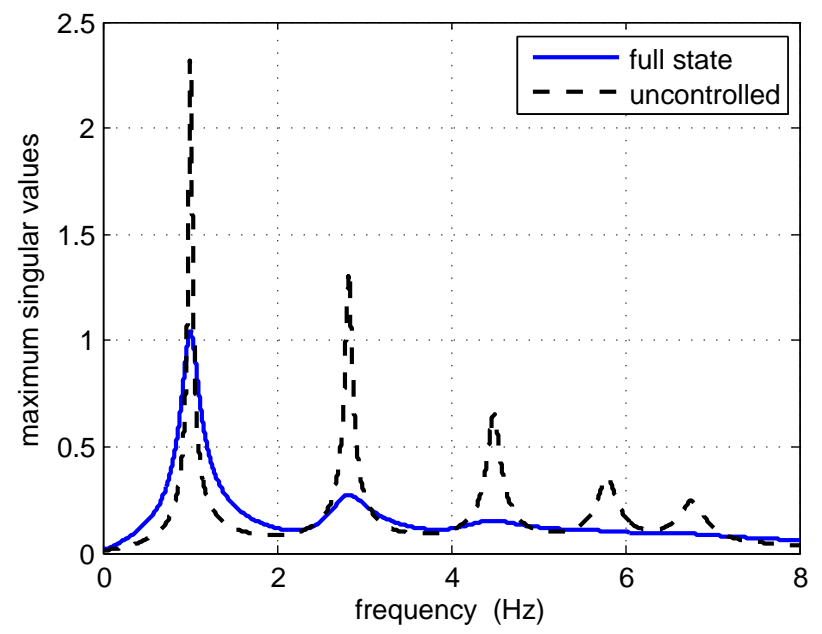

Figure 3: Frequency response corresponding to the optimal state-feedback $H_{\infty}$ controller $\mathbf{u}(t)=\widetilde{\mathbf{G}}_{s} \mathbf{x}(t)$. Maximum singular values of the closed-loop pulse transfer function $\mathbf{T}_{\widetilde{\mathbf{G}}_{s}}(2 \pi f j)$ (solid blue line) and the open-loop transfer function $\mathbf{T}(2 \pi f j$ ) (dashed black line)

the value $\gamma_{G}$ can be obtained as the $H_{\infty}$-norm of $\mathbf{T}_{G}$

$$
\gamma_{G}=\left\|\mathbf{T}_{G}\right\|_{\infty}=\sup _{f} \sigma_{\max }\left[\mathbf{T}_{G}(2 \pi f j)\right]
$$

where $j=\sqrt{-1}, f$ is the frequency in Hertz and $\sigma_{\max }[\cdot]$ denotes the maximum singular value.

Broadly speaking, the controller design consists in computing a control gain matrix $\widetilde{G}$ that produces an asymptotically stable closed-loop matrix $\mathbf{A}_{\widetilde{G}}$ and attains an optimally small $H_{\infty}$-norm $\gamma_{\widetilde{G}}$. It is shown in [13] that these objectives can be achieved by solving the following LMI optimization problem:

$$
\begin{aligned}
& \mathscr{P}_{s}:\left\{\begin{array}{l}
\text { maximize } \eta \\
\text { subject to } \mathbf{X}>0, \eta>0 \text { and the LMI in (32), }
\end{array}\right. \\
& {\left[\begin{array}{cr}
\mathbf{A X}+\mathbf{X A}^{T}+\mathbf{B}_{u} \mathbf{Y}+\mathbf{Y}^{T} \mathbf{B}_{u}^{T}+\eta \mathbf{B}_{w} \mathbf{B}_{w}^{T} & * \\
\mathbf{C}_{z} \mathbf{X}+\mathbf{D}_{z} \mathbf{Y} & -\mathbf{I}
\end{array}\right]<0,}
\end{aligned}
$$

where the matrices $\mathbf{X}$ and $\mathbf{Y}$ are the optimization variables and $*$ represents the transpose of the element in the symmetric position. If the optimization problem $\mathscr{P}_{s}$ attains an optimal value $\widetilde{\eta}_{s}$ for the pair of matrices $\left(\widetilde{\mathbf{X}}_{s}, \widetilde{\mathbf{Y}}_{s}\right)$, then the gain matrix

$$
\widetilde{\mathbf{G}}_{s}=\widetilde{\mathbf{Y}}_{s} \widetilde{\mathbf{X}}_{s}^{-1}
$$

defines a state-feedback controller $\mathbf{u}(t)=\widetilde{\mathbf{G}}_{s} \mathbf{x}(t)$ with an asymptotically stable closed-loop matrix $\mathbf{A}_{\widetilde{G}_{s}}$ and an optimal $H_{\infty}$ norm

$$
\gamma_{\widetilde{G}_{s}}=\left(\widetilde{\eta}_{s}\right)^{-1 / 2} .
$$

By solving the optimization problem $\mathscr{P}_{s}$ with the system matrices $\mathbf{A}, \mathbf{B}_{u}$ and $\mathbf{B}_{w}$ given in (19) and (20), the matrices $\mathbf{C}_{z}$ and $\mathbf{D}_{z}$ defined in (22), and the value

$$
\alpha=10^{-6.3}
$$

we obtain the following state gain matrix:

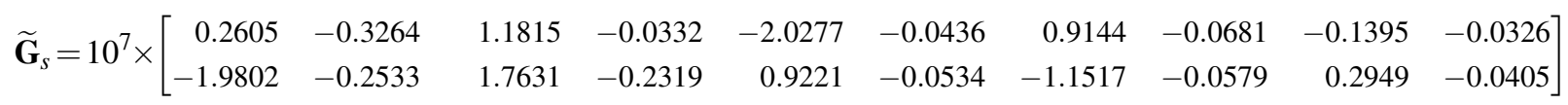

and the optimal $\gamma$-value

$$
\gamma_{\widetilde{G}_{s}}=1.0409
$$




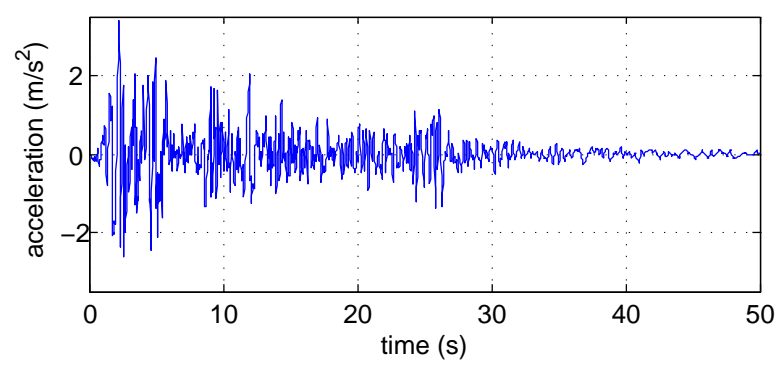

Figure 4: Full scale North-South El Centro 1940 seismic record with an absolute acceleration peak of $3.42 \mathrm{~m} / \mathrm{s}^{2}$

To illustrate the frequency behaviour of the state-feedback controller defined by the gain matrix $\widetilde{\mathbf{G}}_{s}$, the maximum singular values of the closed-loop pulse transfer function

$$
\mathbf{T}_{\widetilde{G}_{s}}(2 \pi f j)=\mathbf{C}_{\widetilde{G}_{s}}\left(2 \pi f j \mathbf{I}-\mathbf{A}_{\widetilde{G}_{s}}\right)^{-1} \mathbf{B}_{w}
$$

and the open-loop pulse transfer function

$$
\mathbf{T}(2 \pi f j)=\mathbf{C}_{z}(2 \pi f j \mathbf{I}-\mathbf{A})^{-1} \mathbf{B}_{w}
$$

are presented in Figure 3. The graphic corresponding to the open-loop transfer function (dashed black line) shows the frequency response characteristics of the uncontrolled structure, where it can be appreciated the structure resonant frequencies located at 1.01, 2.82, 4.49, 5.80 and $6.77 \mathrm{~Hz}$. The graphic corresponding to the closed-loop transfer function $\mathbf{T}_{\widetilde{G}_{s}}(2 \pi f j)$ (solid blue line) clearly shows the ability of the state-feedback $H_{\infty}$ controller to mitigate the structural vibration response at the resonant frequencies. The magnitude of the largest peak in this graphic corresponds to the $\gamma$-value given in (37).

To complement the information provided by the frequency plots, we have also conducted numerical simulations of the structural vibration response using the full scale North-South El Centro 1940 seismic record as ground acceleration input (see Figure 4). The maximum absolute interstory drifts and maximum absolute control efforts corresponding to the ideal state-feedback $H_{\infty}$ controller defined by the control gain matrix $\widetilde{\mathbf{G}}_{s}$ are presented in Figure 5, using a blue line with circles. The maximum absolute interstory drifts corresponding to the uncontrolled structure are also included as a reference, using a black line with rectangles. Looking at the graphics in Figure 5, it can be appreciated that the proposed state-feedback $H_{\infty}$ controller attains a good level of reduction in the interstory drift peak-values with moderate levels of control effort. In what follows, we will assume that $\widetilde{\mathbf{G}}_{s}$ defines a satisfactory state-feedback controller for the structure introduced in Section 2.

Remark 1 According to the lower graphic in Figure 5, the control actions corresponding to the proposed state-feedback controller present peak-values in the range 0.8-1.1 MN. Control forces of this magnitude are commonly used in modern control systems for vibration control of large structures [1,29].

Remark 2 In addition to the state gain matrix $\widetilde{\mathbf{G}}_{s}$, solving the LMI optimization problem $\mathscr{P}_{s}$ given in (31) produces the optimal $X$-matrix

$$
\widetilde{\mathbf{X}}_{s}=\left[\begin{array}{rrrrrrrrrr}
0.0041 & -0.0053 & 0.0001 & -0.0090 & 0.0005 & 0.0189 & 0.0001 & -0.0085 & -0.0002 & 0.0035 \\
-0.0053 & 2.4008 & 0.0207 & -2.3443 & -0.0200 & 0.1274 & 0.0085 & -0.0904 & -0.0020 & -0.1572 \\
0.0001 & 0.0207 & 0.0053 & -0.0113 & 0.0005 & -0.0404 & 0.0001 & 0.0282 & 0.0001 & -0.0030 \\
-0.0090 & -2.3443 & -0.0113 & 4.6285 & 0.0491 & -1.9679 & -0.0246 & -0.3235 & 0.0025 & 0.0444 \\
0.0005 & -0.0200 & 0.0005 & 0.0491 & 0.0044 & -0.0060 & 0.0013 & -0.0320 & 0.0003 & 0.0103 \\
0.0189 & 0.1274 & -0.0404 & -1.9679 & -0.0060 & 3.3677 & 0.0345 & -1.0694 & -0.0083 & -0.3602 \\
0.0001 & 0.0085 & 0.0001 & -0.0246 & 0.0013 & 0.0345 & 0.0047 & -0.0042 & 0.0015 & -0.0169 \\
-0.0085 & -0.0904 & 0.0282 & -0.3235 & -0.0320 & -1.0694 & -0.0042 & 2.8709 & 0.0172 & -0.8863 \\
-0.0002 & -0.0020 & 0.0001 & 0.0025 & 0.0003 & -0.0083 & 0.0015 & 0.0172 & 0.0049 & -0.0038 \\
0.0035 & -0.1572 & -0.0030 & 0.0444 & 0.0103 & -0.3602 & -0.0169 & -0.8863 & -0.0038 & 2.8991
\end{array}\right] .
$$

In the next sections, we will see that this matrix plays an important role in the definition of the LMI optimization problems used to compute the proposed output-feedback controllers with partial state information. 

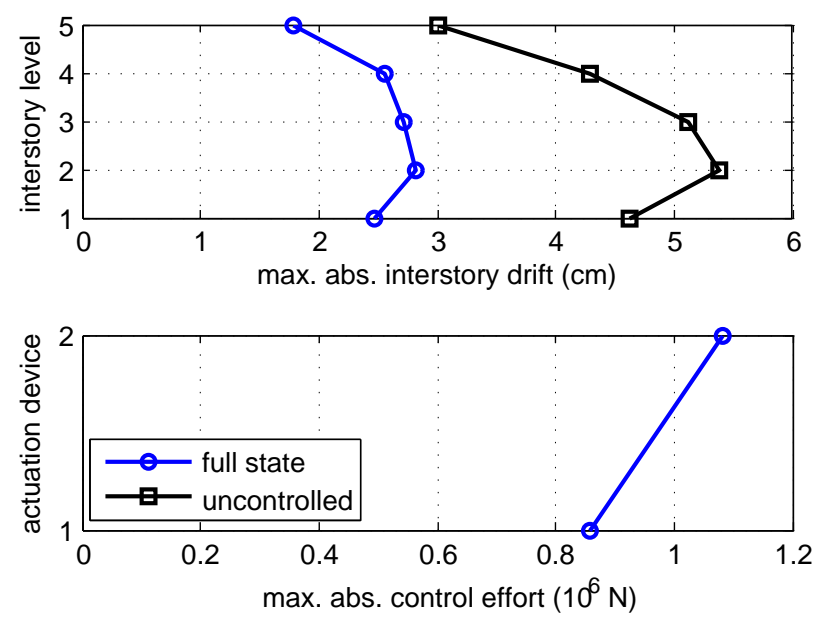

Figure 5: Maximum absolute interstory drifts and maximum absolute control efforts corresponding to the ideal state-feedback $H_{\infty}$ controller defined by the control gain matrix $\widetilde{\mathbf{G}}_{s}$ (blue line with circles). The full scale North-South El Centro 1940 seismic record has been used as ground acceleration disturbance

Remark 3 In this kind of control problems, interstory drift peak-values are typically in the order of $10^{-2} \mathrm{~m}$, interstory velocity peak-values are in the order of $10^{-1} \mathrm{~m} / \mathrm{s}$, and the control force peak-values are in the order of $10^{6} \mathrm{~N}$. Consequently an $\alpha$-value of the order $10^{-6}$ or $10^{-7}$ is naturally required to introduce a proper scaling of the magnitudes involved in the controlled-output defined in (21) and (22). For the same reason, a scaling factor of the order $10^{6}$ or $10^{7}$ appears naturally in the control gain matrices. A particular $\alpha$-value can be obtained by considering the frequency response plots corresponding to the open-loop and controlled systems and the magnitude of the elements in the control gain matrix. Specifically, the $\alpha$-value in (35) has been selected based on the frequency-response plots in Figure 3 and the magnitude of the elements in the control matrix (36). The good behaviour of the obtained controller has been further checked by means of the time-response peak-value plots in Figure 5. It should be noted that no additional parameter or initialization values need to be tuned in the proposed controller design methodology.

\section{$4 H_{\infty}$ controller with neighbouring state information}

Now, let us assume that the information available for feedback purposes is a vector of measured outputs $\mathbf{y}(t)$ that can be expressed as linear combinations of the states in the form

$$
\mathbf{y}(t)=\mathbf{C}_{y} \mathbf{x}(t)
$$

In this case, we are interested in designing static output-feedback controllers

$$
\mathbf{u}(t)=\mathbf{K} \mathbf{y}(t)
$$

which can compute the control actions $\mathbf{u}(t)$ from the measured outputs $\mathbf{y}(t)$ by means of a simple matrix product. By considering (9), (41) and (42), we obtain the closed-loop system

$$
\left\{\begin{array}{l}
\dot{\mathbf{x}}(t)=\mathbf{A}_{K} \mathbf{x}(t)+\mathbf{B}_{w} w(t), \\
\mathbf{z}_{K}(t)=\mathbf{C}_{K} \mathbf{x}(t),
\end{array}\right.
$$

with

$$
\mathbf{A}_{K}=\mathbf{A}+\mathbf{B}_{u} \mathbf{K C}_{y}, \quad \mathbf{C}_{K}=\mathbf{C}_{z}+\mathbf{D}_{z} \mathbf{K C}_{y} .
$$

The $H_{\infty}$-norm of the output-feedback controller defined by the output gain matrix $\mathbf{K}$ is the value

$$
\gamma_{K}=\sup _{\|w\|_{2} \neq 0} \frac{\left\|\mathbf{z}_{K}\right\|_{2}}{\|w\|_{2}} .
$$




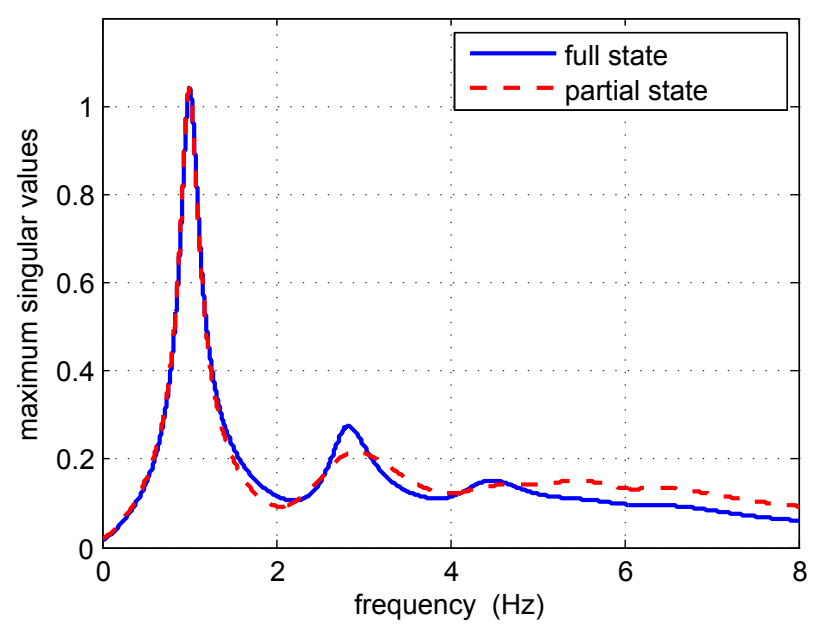

Figure 6: Frequency response corresponding to the output-feedback $H_{\infty}$ controller with partial state information $\mathbf{u}(t)=$ $\widetilde{\mathbf{K}}_{\mathrm{I}} \mathbf{y}_{\mathrm{I}}(t)$. Maximum singular values of the closed-loop pulse transfer functions $\mathbf{T}_{\widetilde{K}_{\mathrm{I}}}(2 \pi f j)$ (dashed red line) and $\mathbf{T}_{\widetilde{G}_{s}}(2 \pi f j$ ) (solid blue line)

Using the closed-loop transfer function from the disturbance input $w(t)$ to the controlled output $\mathbf{z}_{K}(t)$

$$
\mathbf{T}_{K}(s)=\mathbf{C}_{K}\left(s \mathbf{I}-\mathbf{A}_{K}\right)^{-1} \mathbf{B}_{w}
$$

the value $\gamma_{K}$ can be obtained as the $H_{\infty}$-norm of $\mathbf{T}_{K}$

$$
\gamma_{K}=\left\|\mathbf{T}_{K}\right\|_{\infty}=\sup _{f} \sigma_{\max }\left[\mathbf{T}_{K}(2 \pi f j)\right]
$$

The output-feedback $H_{\infty}$ design objective consists in obtaining a gain matrix $\widetilde{\mathbf{K}}$ that produces an asymptotically stable closedloop matrix $\mathbf{A}_{\widetilde{K}}$ and, simultaneously, attains an optimally small $\gamma$-value $\gamma_{\widetilde{K}}$. In this case, however, it should be highlighted that obtaining an optimal matrix $\widetilde{\mathbf{K}}$ can involve serious computational difficulties [17-20].

According to the design method presented in [21], a suboptimal gain matrix $\widetilde{\mathbf{K}}$ can be effectively computed by considering the state-feedback LMI optimization problem $\mathscr{P}_{s}$ in (31) and the following transformations of the LMI variables $\mathbf{X}$ and $\mathbf{Y}$ :

$$
\mathbf{X}=\mathbf{Q} \mathbf{X}_{Q} \mathbf{Q}^{T}+\mathbf{R} \mathbf{X}_{R} \mathbf{R}^{T}, \quad \mathbf{Y}=\mathbf{Y}_{R} \mathbf{R}^{T}
$$

which introduce, as new LMI variables, two symmetric matrices $\mathbf{X}_{Q}$ and $\mathbf{X}_{R}$ and a rectangular matrix $\mathbf{Y}_{R}$. The transformations also involve two constant matrices: a matrix $\mathbf{Q}$, whose columns contain a basis of $\operatorname{Ker}\left(\mathbf{C}_{y}\right)$ and a matrix $\mathbf{R}$ with the following form:

$$
\mathbf{R}=\mathbf{C}_{y}^{\dagger}+\mathbf{Q} \mathbf{L}
$$

where

$$
\mathbf{C}_{y}^{\dagger}=\mathbf{C}_{y}^{T}\left(\mathbf{C}_{y} \mathbf{C}_{y}^{T}\right)^{-1}
$$

is the Moore-Penrose pseudoinverse of $\mathbf{C}_{y}$, and $\mathbf{L}$ is an arbitrary constant matrix that allows defining LMI variables transformations with different degrees of computational effectiveness. Recently, the following advanced $L$-matrix choice has been proposed in [22]:

$$
\widetilde{\mathbf{L}}=\mathbf{Q}^{\dagger} \widetilde{\mathbf{X}}_{s} \mathbf{C}_{y}^{T}\left(\mathbf{C}_{y} \widetilde{\mathbf{X}}_{s} \mathbf{C}_{y}^{T}\right)^{-1}
$$

where

$$
\mathbf{Q}^{\dagger}=\left(\mathbf{Q}^{T} \mathbf{Q}\right)^{-1} \mathbf{Q}^{T}
$$

is the Moore-Penrose pseudoinverse of $\mathbf{Q}$ and $\widetilde{\mathbf{X}}_{s}$ is the optimal $X$-matrix of the state-feedback optimization problem $\mathscr{P}_{s}$. By substituting the transformations given in (48) into the LMI presented in (32), we obtain the following LMI optimization 
problem:

$$
\begin{gathered}
\mathscr{P}_{o}:\left\{\begin{array}{lr}
\text { maximize } \eta \\
\text { subject to } \mathbf{X}_{Q}>0, \mathbf{X}_{R}>0, \eta>0 \text { and the LMI in (54), }
\end{array}\right. \\
{\left[\begin{array}{cr}
\mathbf{A} \mathbf{Q} \mathbf{X}_{Q} \mathbf{Q}^{T}+\mathbf{Q} \mathbf{X}_{Q} \mathbf{Q}^{T} \mathbf{A}^{T}+\mathbf{A} \mathbf{R} \mathbf{X}_{R} \mathbf{R}^{T}+\mathbf{R} \mathbf{X}_{R} \mathbf{R}^{T} \mathbf{A}^{T}+\mathbf{B}_{u} \mathbf{Y}_{R} \mathbf{R}^{T}+\mathbf{R} \mathbf{Y}_{R}^{T} \mathbf{B}_{u}^{T}+\eta \mathbf{B}_{w} \mathbf{B}_{w}^{T} & * \\
\mathbf{C}_{z} \mathbf{Q} \mathbf{X}_{Q} \mathbf{Q}^{T}+\mathbf{C}_{z} \mathbf{R} \mathbf{X}_{R} \mathbf{R}^{T}+\mathbf{D}_{z} \mathbf{Y}_{R} \mathbf{R}^{T} & -\mathbf{I}
\end{array}\right]<0,}
\end{gathered}
$$

where $\mathbf{X}_{Q}, \mathbf{X}_{R}$ and $\mathbf{Y}_{R}$ are the optimization variables. If an optimal value $\widetilde{\eta}_{o}$ is attained in $\mathscr{P}_{o}$ for the triplet $\left(\widetilde{\mathbf{X}}_{Q}, \widetilde{\mathbf{X}}_{R}, \widetilde{\mathbf{Y}}_{R}\right)$, then the output gain matrix $\widetilde{\mathbf{K}}$ can be written in the form

$$
\widetilde{\mathbf{K}}=\widetilde{\mathbf{Y}}_{R}\left(\widetilde{\mathbf{X}}_{R}\right)^{-1},
$$

and the corresponding $\gamma$-value satisfies

$$
\gamma_{\widetilde{K}} \leq\left(\widetilde{\eta}_{o}\right)^{-1 / 2} .
$$

Now, for the structure model presented in Section 2, let us assume that the information available for feedback purposes is the output vector

$$
\mathbf{y}_{\mathrm{I}}(t)=\left[r_{1}(t), v_{1}(t), r_{2}(t), v_{2}(t)\right]^{T},
$$

which contains the state variables corresponding to the instrumented levels. By taking the matrix

$$
\left(\mathbf{C}_{y}\right)_{\mathrm{I}}=\left[\begin{array}{llllllllll}
1 & 0 & 0 & 0 & 0 & 0 & 0 & 0 & 0 & 0 \\
0 & 1 & 0 & 0 & 0 & 0 & 0 & 0 & 0 & 0 \\
0 & 0 & 1 & 0 & 0 & 0 & 0 & 0 & 0 & 0 \\
0 & 0 & 0 & 1 & 0 & 0 & 0 & 0 & 0 & 0
\end{array}\right],
$$

the measured output vector $\mathbf{y}_{\mathrm{I}}(t)$ can be expressed in the form

$$
\mathbf{y}_{\mathrm{I}}(t)=\left(\mathbf{C}_{y}\right)_{\mathrm{I}} \mathbf{x}(t),
$$

where $\mathbf{x}(t)$ is the state vector defined in (8). In this case, we are interested in designing a static output-feedback $H_{\infty}$ controller

$$
\mathbf{u}(t)=\widetilde{\mathbf{K}}_{\mathrm{I}} \mathbf{y}_{\mathrm{I}}(t),
$$

which allows computing the control actions $\mathbf{u}(t)$ from the restricted neighbouring state information provided by the measured output vector $\mathbf{y}_{\mathrm{I}}(t)$. To this end, we consider the $Q$-matrix

$$
\mathbf{Q}_{I}=\left[\begin{array}{llllll}
0 & 0 & 0 & 0 & 0 & 0 \\
0 & 0 & 0 & 0 & 0 & 0 \\
0 & 0 & 0 & 0 & 0 & 0 \\
0 & 0 & 0 & 0 & 0 & 0 \\
1 & 0 & 0 & 0 & 0 & 0 \\
0 & 1 & 0 & 0 & 0 & 0 \\
0 & 0 & 1 & 0 & 0 & 0 \\
0 & 0 & 0 & 1 & 0 & 0 \\
0 & 0 & 0 & 0 & 1 & 0 \\
0 & 0 & 0 & 0 & 0 & 1
\end{array}\right]
$$

and, by substituting $\left(\mathbf{C}_{y}\right)_{\mathrm{I}}, \mathbf{Q}_{\mathrm{I}}$ and the matrix $\widetilde{\mathbf{X}}_{s}$ given in (40) into the expression (51), we obtain the following $L$-matrix:

$$
\widetilde{\mathbf{L}}_{\mathrm{I}}=\left[\begin{array}{rrrr}
0.1606 & 0.0040 & 0.1026 & 0.0132 \\
2.4151 & -0.6121 & -6.8346 & -0.7471 \\
-0.0059 & -0.0035 & 0.0113 & -0.0071 \\
-3.1727 & -0.3111 & 6.1254 & -0.2187 \\
-0.0462 & -0.0013 & 0.0227 & -0.0001 \\
0.6361 & -0.1026 & -0.2637 & -0.0418
\end{array}\right],
$$




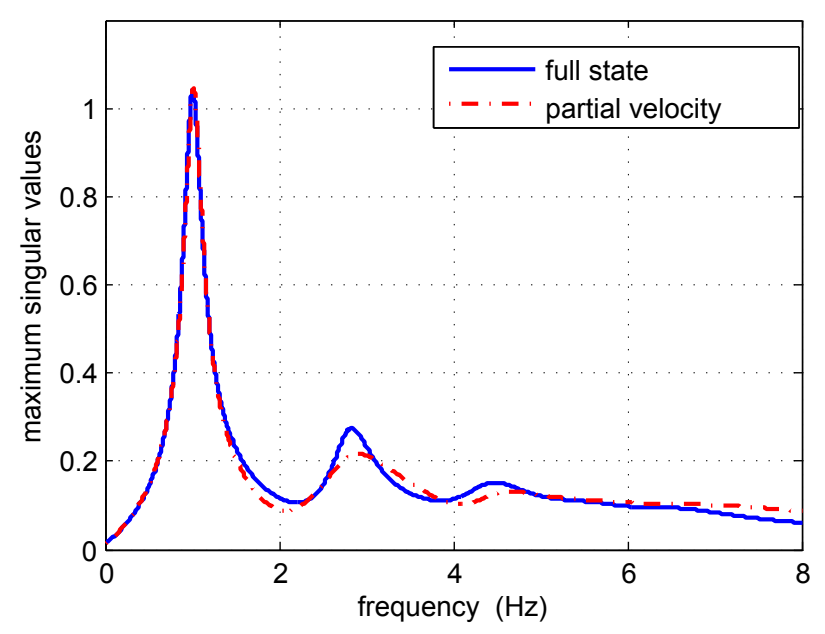

Figure 7: Frequency response corresponding to the output-feedback $H_{\infty}$ controller with partial velocity information $\mathbf{u}(t)=$ $\widetilde{\mathbf{K}}_{\mathrm{II}} \mathbf{y}_{\mathrm{II}}(t)$. Maximum singular values of the pulse transfer functions $\mathbf{T}_{\widetilde{\mathbf{K}}_{\mathrm{II}}}(2 \pi f j)$ (dash-dotted red line) and $\mathbf{T}_{\widetilde{G}_{s}}(2 \pi f j)($ solid blue line)

which, considering equation (49), produces the $R$-matrix

$$
\mathbf{R}_{\mathrm{I}}=\left[\begin{array}{c}
\mathbf{I}_{4} \\
\widetilde{\mathbf{L}}_{\mathrm{I}}
\end{array}\right]
$$

Next, by solving the output-feedback LMI optimization problem $\mathscr{P}_{o}$ in (53) with the matrices $\mathbf{Q}_{\mathrm{I}}$ and $\mathbf{R}_{\mathrm{I}}$ given in (61) and (63), respectively, and the same matrices $\mathbf{A}, \mathbf{B}_{u}, \mathbf{B}_{w}, \mathbf{C}_{z}$ and $\mathbf{D}_{z}$ used in the state-feedback LMI optimization problem $\mathscr{P}_{s}$, we obtain the following output gain matrix:

$$
\widetilde{\mathbf{K}}_{\mathrm{I}}=10^{7} \times\left[\begin{array}{rrrr}
0.6702 & -0.5603 & -0.2790 & -0.0308 \\
-2.4526 & -0.3939 & 2.4081 & -0.2221
\end{array}\right]
$$

with an associated $\gamma$-value that satisfies

$$
\gamma_{\widetilde{K}_{\mathrm{I}}} \leq 1.0410
$$

Comparing the upper bound in (65) with the gamma value in (37), corresponding to the optimal state-feedback $H_{\infty}$ controller, we can see that the $H_{\infty}$ controller with neighbouring state information defined by the output matrix $\widetilde{\mathbf{K}}_{\mathrm{I}}$ is practically optimal. In fact, by using the procedure indicated in Remark 6 , we obtain the value $\gamma_{\widetilde{K}_{\mathrm{I}}}=1.0410$. In Figure 6 , we present the maximum singular values of the closed-loop pulse transfer function

$$
\mathbf{T}_{\widetilde{K}_{\mathrm{I}}}(2 \pi f j)=\mathbf{C}_{\widetilde{K}_{\mathrm{I}}}\left(2 \pi f j \mathbf{I}-\mathbf{A}_{\widetilde{K}_{\mathrm{I}}}\right)^{-1} \mathbf{B}_{w}
$$

associated to the output-feedback controller $\mathbf{u}(t)=\widetilde{\mathbf{K}}_{\mathrm{I}} \mathbf{y}_{\mathrm{I}}(t)$ (dashed red line) and the maximum singular values of the closedloop pulse transfer function $\mathbf{T}_{\widetilde{G}_{s}}(2 \pi f j)$ associated to the optimal state-feedback controller $\mathbf{u}(t)=\widetilde{\mathbf{G}}_{s} \mathbf{x}(t)$ (solid blue line). The graphics presented in Figures 3 and 6 show the notable similarity of the frequency responses produced by the proposed static output-feedback controller and the optimal state-feedback controller.

Remark 4 In Figure 6 and Figure 7, the open-loop response has not been included to provide a more detailed view of the controlled responses. To obtain a proper idea of the differences' magnitude, the plots in these figures should be compared with the open-loop frequency response displayed in Figure 3. Note that in all these figures, the solid blue line represents the frequency response of the optimal state-feedback $H_{\infty}$ controller $\mathbf{u}(t)=\widetilde{\mathbf{G}}_{s} \mathbf{x}(t)$ computed in Section 3.

\section{$5 \quad H_{\infty}$ controller with neighbouring velocity information}

To illustrate the flexibility of the proposed controller design methodology, we consider again the structure model presented in Section 2 but, in this case, we assume that the information available for feedback purposes consists only in the interstory 
velocities $v_{1}(t)$ and $v_{2}(t)$. Now, we have the output vector

$$
\mathbf{y}_{\mathrm{II}}(t)=\left[v_{1}(t), v_{2}(t)\right]^{T},
$$

which can be expressed in the form

$$
\mathbf{y}_{\mathrm{II}}(t)=\left(\mathbf{C}_{y}\right)_{\mathrm{II}} \mathbf{x}(t)
$$

with the output matrix

$$
\left(\mathbf{C}_{y}\right)_{\mathrm{II}}=\left[\begin{array}{llllllllll}
0 & 1 & 0 & 0 & 0 & 0 & 0 & 0 & 0 & 0 \\
0 & 0 & 0 & 1 & 0 & 0 & 0 & 0 & 0 & 0
\end{array}\right] .
$$

In this case, we are interested in designing a static output-feedback $H_{\infty}$ controller

$$
\mathbf{u}(t)=\widetilde{\mathbf{K}}_{\mathrm{II}} \mathbf{y}_{\mathrm{II}}(t),
$$

which allows computing the control actions $\mathbf{u}(t)$ from the restricted neighbouring interstory velocity information provided by the output vector $\mathbf{y}_{\mathrm{II}}(t)$. To this end, we proceed as we did in the previous section and we solve the output-feedback LMI optimization problem $\mathscr{P}_{o}$ in (53) with the same matrices $\mathbf{A}, \mathbf{B}_{u}, \mathbf{B}_{w}, \mathbf{C}_{z}$ and $\mathbf{D}_{z}$ used in the state-feedback LMI optimization problem $\mathscr{P}_{s}$, the $Q$-matrix

$$
\mathbf{Q}_{\mathrm{II}}=\left[\begin{array}{llllllll}
0 & 1 & 0 & 0 & 0 & 0 & 0 & 0 \\
0 & 0 & 0 & 0 & 0 & 0 & 0 & 0 \\
1 & 0 & 0 & 0 & 0 & 0 & 0 & 0 \\
0 & 0 & 0 & 0 & 0 & 0 & 0 & 0 \\
0 & 0 & 1 & 0 & 0 & 0 & 0 & 0 \\
0 & 0 & 0 & 1 & 0 & 0 & 0 & 0 \\
0 & 0 & 0 & 0 & 1 & 0 & 0 & 0 \\
0 & 0 & 0 & 0 & 0 & 1 & 0 & 0 \\
0 & 0 & 0 & 0 & 0 & 0 & 1 & 0 \\
0 & 0 & 0 & 0 & 0 & 0 & 0 & 1
\end{array}\right]
$$

and the $R$-matrix

$$
\mathbf{R}_{\mathrm{II}}=\left[\begin{array}{rr}
-0.0081 & -0.0060 \\
1.0000 & 0.0000 \\
0.0124 & 0.0038 \\
0.0000 & 1.0000 \\
0.0040 & 0.0126 \\
-0.7164 & -0.7880 \\
-0.0033 & -0.0070 \\
-0.2095 & -0.1760 \\
-0.0006 & 0.0002 \\
-0.1110 & -0.0466
\end{array}\right] .
$$

As a result, we obtain the output gain matrix

$$
\widetilde{\mathbf{K}}_{\mathrm{II}}=10^{6} \times\left[\begin{array}{ll}
-5.4203 & -0.2906 \\
-3.6754 & -2.8287
\end{array}\right]
$$

and the following upper bound for the associated $\gamma$-value:

$$
\gamma_{\widetilde{K}_{\text {II }}} \leq 1.0469
$$

Comparing the bound in (74) with the optimal gamma value in (37), we can see that the $\gamma$-value increment produced by the proposed velocity-feedback $H_{\infty}$ controller is inferior to $0.6 \%$. The maximum singular values of the closed-loop pulse transfer function

$$
\mathbf{T}_{\widetilde{K}_{\text {II }}}(2 \pi f j)=\mathbf{C}_{\widetilde{K}_{\text {II }}}\left(2 \pi f j \mathbf{I}-\mathbf{A}_{\widetilde{K}_{\text {II }}}\right)^{-1} \mathbf{B}_{w}
$$



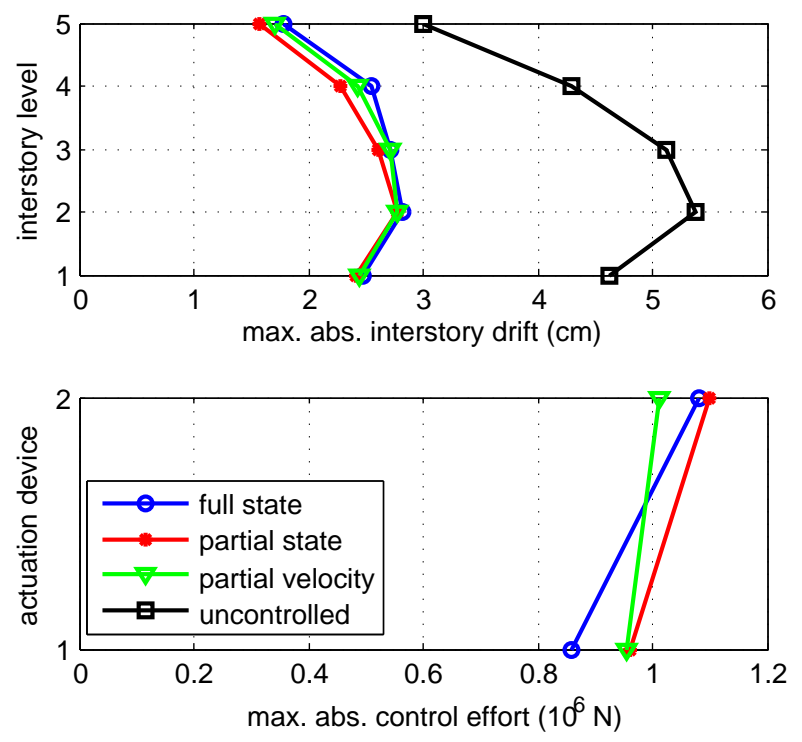

Figure 8: Maximum absolute interstory drifts and maximum absolute control efforts corresponding to the optimal statefeedback controller $\mathbf{u}(t)=\widetilde{\mathbf{G}}_{s} \mathbf{x}(t)$ (blue line with circles), the output-feedback controller $\mathbf{u}(t)=\widetilde{\mathbf{K}}_{\mathrm{I}} \mathbf{y}_{\mathrm{I}}(t)$ (red line with asterisks), the velocity-feedback controller $\mathbf{u}(t)=\widetilde{\mathbf{K}}_{\mathrm{II}} \mathbf{y}_{\mathrm{II}}(t)$ (green line with triangles), and the uncontrolled structure (black line with rectangles). The full scale North-South El Centro 1940 seismic record has been used as ground acceleration disturbance

are displayed in Figure 7 using a dash-dotted red line. The solid blue line in the figure represents the maximum singular values of the closed-loop pulse transfer function $\mathbf{T}_{\widetilde{G}_{s}}(2 \pi f j)$ associated to the optimal state-feedback controller $\mathbf{u}(t)=\widetilde{\mathbf{G}}_{s} \mathbf{x}(t)$. Looking at the graphics in Figure 7, it can be appreciated that the frequency response produced by the velocity-feedback controller $\mathbf{u}(t)=\widetilde{\mathbf{K}}_{\mathrm{II}} \mathbf{y}_{\mathrm{II}}(t)$ is very similar to the frequency response corresponding to the optimal state-feedback controller. Moreover, considering the graphics in Figure 6, it can also be observed that the overall frequency response of the proposed velocity-feedback controller is slightly better than the frequency response of the output-feedback controller with partial state information $\mathbf{u}(t)=\widetilde{\mathbf{K}}_{\mathrm{I}} \mathbf{y}_{\mathrm{I}}(t)$.

Finally, to gain a better insight into the behaviour of the proposed output-feedback controllers and to complement the information provided by the frequency plots, numerical simulations of the structural vibration response have been conducted using the full scale North-South El Centro 1940 seismic record as ground acceleration input. The maximum absolute interstory drifts and maximum absolute control efforts corresponding to the different control configurations are presented in Figure 8, using the following colours and symbols: (i) the blue line with circles corresponds to the ideal state-feedback $H_{\infty}$ controller $\mathbf{u}(t)=\widetilde{\mathbf{G}}_{s} \mathbf{x}(t)$ (denoted as "full state" in the legend), (ii) the red line with asterisks represents the output-feedback controller with partial state information $\mathbf{u}(t)=\widetilde{\mathbf{K}}_{\mathrm{I}} \mathbf{y}_{\mathrm{I}}(t)$ (denoted as "partial state" in the legend), (iii) the green line with triangles displays the data of the velocity-feedback controller $\mathbf{u}(t)=\widetilde{\mathbf{K}}_{\mathrm{II}} \mathbf{y}_{\mathrm{II}}(t)$ (denoted as "partial velocity" in the legend), and (iv) the black line with rectangles presents the data corresponding to the uncontrolled structure response.

A quick look at the graphics in Figure 8 indicates that the overall behaviour exhibited by the three controllers is very similar, attaining levels of reduction in the interstory drift peak-values of about $40 \%-50 \%$ with respect to the uncontrolled response. In all the cases, the control-effort peak-values are in the range $0.8-1.1 \mathrm{MN}$. In a more detailed inspection, it can be appreciated that the most balanced behaviour corresponds to the velocity-feedback controller defined by the output gain matrix $\widetilde{\mathbf{K}}_{\mathrm{II}}$. Despite the severe constraints on feedback information, the controller $\mathbf{u}(t)=\widetilde{\mathbf{K}}_{\mathrm{II}} \mathbf{y}_{\mathrm{II}}(t)$ achieves the same levels of reduction in the interstory drift peak-values as the ideal state-feedback controller with full state information, producing control-effort peak-values of about $0.9-1.0 \mathrm{MN}$.

Remark 5 The matrix $\mathbf{Q}_{\text {II }}$ presented in (71) has been computed with the Matlab command null(). Obviously, other choices of this matrix are also possible. The particular matrix $\mathbf{Q}_{\text {II }}$ has been selected to improve the consistence with the 
Matlab results. The matrix $\mathbf{R}_{\mathrm{II}}$ in (72) has been obtained using the following $L$-matrix:

$$
\widetilde{\mathbf{L}}_{\mathrm{II}}=\left[\begin{array}{rr}
0.0124 & 0.0038 \\
-0.0081 & -0.0060 \\
0.0040 & 0.0126 \\
-0.7164 & -0.7880 \\
-0.0033 & -0.0070 \\
-0.2095 & -0.1760 \\
-0.0006 & 0.0002 \\
-0.1110 & -0.0466
\end{array}\right]
$$

which has been computed by substituting $\left(\mathbf{C}_{y}\right)_{\mathrm{II}}, \mathbf{Q}_{\mathrm{II}}$ and $\widetilde{\mathbf{X}}_{s}$ into the expression (51).

Remark 6 It should be noted that the value provided in (74) is just an upper bound. The actual $H_{\infty}$-norm of the controller $\mathbf{u}(t)=\widetilde{\mathbf{K}}_{\mathrm{II}} \mathbf{y}_{\mathrm{II}}(t)$ can be obtained by solving the optimization problem

$$
\gamma_{\widetilde{K}_{\mathrm{II}}}=\left\|\mathbf{T}_{\widetilde{K}_{\mathrm{II}}}\right\|_{\infty}=\sup _{f} \sigma_{\max }\left[\mathbf{T}_{\widetilde{K}_{\mathrm{II}}}(2 \pi f j)\right]
$$

which produces the $\gamma$-value

$$
\gamma_{\widetilde{K}_{\mathrm{II}}}=1.0458
$$

corresponding to the largest peak-value of the dash-dotted red line in Figure 7.

Remark 7 The underlying idea in the design of the controllers (60) and (70) consists in obtaining a static output-feedback controller $\mathbf{u}(t)=\widetilde{\mathbf{K}} \mathbf{y}(t)$ that satisfies $\gamma_{\widetilde{K}} \approx \gamma_{\widetilde{G}_{s}}$. It has been observed in practice that, frequently, the behaviour of static outputfeedback controllers with an almost optimal $\gamma$-value is similar to the behaviour exhibited by the optimal state-feedback controller. Considering the $\gamma$-values given in (37), (65) and (78), the frequency plots displayed in Figures 6 and 7, and the peak-value plots presented in Figure 8, it becomes apparent that this heuristic principle holds for the controllers with partial state information computed in Sections 4 and 5.

Remark 8 In the $H_{\infty}$ approach, the optimality criterion is formulated in terms of minimizing the associated $\gamma$-value. Nevertheless, it should be noted that a smaller $\gamma$-value does not necessarily imply a uniformly better performance over the complete frequency range or uniformly higher levels of reduction in the interstory drift peak-values. Thus, for example, from the $\gamma$-values in (65) and (78), we have the inequality $\gamma_{\widetilde{K}_{\mathrm{I}}}<\gamma_{\widetilde{K}_{\mathrm{II}}}$. However, looking at the graphics in Figures 6 and 7 , it can be clearly appreciated that the controller $\mathbf{u}(t)=\widetilde{\mathbf{K}}_{\mathrm{II}} \mathbf{y}_{\mathrm{II}}(t)$ has a better behaviour than the controller $\mathbf{u}(t)=\widetilde{\mathbf{K}}_{\mathrm{I}} \mathbf{y}_{\mathrm{I}}(t)$ in the frequency range $5-8 \mathrm{~Hz}$.

Remark 9 Design strategies for structured controllers are commonly based on iterative or metaheuristic methods, which aim at producing an acceptable suboptimal solution by solving a sequence of optimization problems. The practical application of such design procedures usually requires determining a suitable set of initialization values and/or algorithm parameters. Additionally, a non-constrained controller is frequently computed to assess the degree of optimality of the obtained solutions. Note that, in the proposed design strategy, the solution is attained after solving two standard LMI optimization problems, the $\alpha$-value of the controlled-output definition in (22) is the only parameter that needs to be adjusted, and the full-state controller designed in the initial step constitutes a natural reference for the performance assessment of the suboptimal structured controllers. Moreover, the $\alpha$-value has a clear meaning in the problem and can be properly adjusted as indicated in Remark 3 .

\section{Conclusions and future directions}

In this paper, a new line of solution to the problem of designing optimal static controllers for vibrational protection of large structures with multiple actuation devices and restricted neighbouring state information has been presented. The proposed design strategy is based on recent advances in static output-feedback control and allows obtaining effective controllers by 
using standard LMI optimization tools. To illustrate the main elements of the new approach, two different $H_{\infty}$ controllers with restricted state information have been computed for the seismic protection of a five-story building with incomplete actuation system. The behaviour of these controllers confirms the effectiveness and flexibility of the new approach and clearly indicates that additional research efforts should be focussed on extending this design strategy to more complex control scenarios that admit a state-feedback LMI-based formulation. Specifically, topics of particular interest are structural vibration control with limited frequency domain [30-32], uncertain and time-delay systems [33-36], vibration control of multi-structure systems [37-39] and networked control strategies [40-42].

\section{Acknowledgments}

This work was partially supported by the following funding sources: the Spanish Ministry of Economy and Competitiveness through the Grant DPI2012-32375/FEDER; the EEA Financial Mechanism through a Grant from Iceland, Liechtenstein and Norway operated by the Universidad Complutense de Madrid; and the Norwegian Center of Offshore Wind Energy (NORCOWE) through the Grant 193821/S60 from the Research Council of Norway (RCN). NORCOWE is a consortium with partners from industry and science, hosted by Christian Michelsen Research.

\section{References}

[1] Spencer B.F., Nagarajaiah S.: 'State of the art of structural control', J. Struct. Eng., 2003, 129, (7), pp. 845-856

[2] Li H., Huo L.: 'Advances in structural control in civil engineering in China', Math. Probl. Eng., 2010, Article ID 936081, pp. 1-23

[3] Thenozhi S., Yu W.: 'Advances in modeling and vibration control of building structures', Annu. Rev. Control, 2013, 37, (2), pp. 346-364

[4] Thenozhi S., Yu W.: 'Stability analysis of active vibration control of building structures using PD/PID control', Eng. Struct., 2014, 81, pp. 208-218

[5] Iuliis M.D., Faella C.: 'Effectiveness analysis of a semiactive base isolation strategy using information from an earlywarning network', Eng. Struct., 2013, 52, pp. 518-535

[6] Oliveira F., Morais P., Suleman A.: 'Predictive control for earthquake response mitigation of buildings using semiactive fluid dampers', Shock Vib., 2014, Article ID 670683, pp. 1-14.

[7] Zhang C., Ou J.: 'Modeling and dynamical performance of the electromagnetic mass driver system for structural vibration control', Eng. Struct., 2015, 82, pp. 93-103

[8] Lei Y., Wu D.T., Liu L.J.: 'A decentralized structural control algorithm with application to the benchmark control problem for seismically excited buildings', Struct. Control Hlth., 2013, 20, (9), pp. 1211-1225

[9] Bitaraf M., Hurlebaus S.: 'Semi-active adaptive control of seismically excited 20-story nonlinear building', Eng. Struct., 2013, 56, pp. 2107-2118

[10] Xiang P., Nishitani A.: 'Seismic vibration control of building structures with multiple tuned mass damper floors integrated', Earthq. Eng. Struct. D., 2014, 43, (6), pp. 909-925

[11] Lynch J.P., Wang Y., Swartz R.A., et al.: 'Implementation of a closed-loop structural control system using wireless sensor networks', Struct. Control Hlth., 2008, 15, (4), pp. 518-539

[12] Wang Y:: 'Time-delayed dynamic output feedback $H_{\infty}$ controller design for civil structures: A decentralized approach through homotopic transformation', Struct. Control Hlth., 2011, 18, (2), pp. 121-139 
[13] Boyd S., Ghaoui L.E., Feron E., Balakrishnan V.: 'Linear Matrix Inequalities in System and Control Theory' (SIAM Studies in Applied Mathematics, Philadelphia, 1994)

[14] Wang Y., Lynch J.P., Law K.H.: 'Decentralized $H_{\infty}$ controller design for large-scale civil structures', Earthq. Eng. Struct. D., 2009, 38, (3), pp. 377-401

[15] Rubió-Massegú J., Palacios-Quiñonero F., Rossell J.M.: 'Decentralized static output-feedback $H_{\infty}$ controller design for buildings under seismic excitation', Earthq. Eng. Struct. D., 2012, 41, (7), pp. 1199-1205

[16] Palacios-Quiñonero F., Rubió-Massegú J., Rossell J.M., et al.: 'Discrete-time multioverlapping controller design for structural vibration control of tall buildings under seismic excitation', Math. Probl. Eng., 2012, Article ID 636878, pp. 1-20

[17] Moerder D.D., Calise A.J.: 'Convergence of a numerical algorithm for calculating optimal output feedback gains', IEEE T. Automat. Contr., 1985, 30, (9), pp. 900-903

[18] Syrmos V.L., Abdallah C.T., Dorato P., et al.: 'Static output feedback - A survey', Automatica, 1997, 33, (2), pp. 125-137

[19] Cao Y.Y., Lam J., Sun Y.X.: 'Static output feedback stabilization: An ILMI approach', Automatica, 1998, 34, (12), pp. $1641-1645$

[20] Shu Z., Lam J.: 'An augmented system approach to static output-feedback stabilization with $H_{\infty}$ performance for continuous-time plants’, Int. J. Robust Nonlin., 2009, 19, (7), pp. 768-785

[21] Rubió-Massegú J., Rossell J.M., Karimi H.R., et al.: 'Static output-feedback control under information structure constraints', Automatica, 2013, 49, (1), pp. 313-316

[22] Palacios-Quiñonero F., Rubió-Massegú J., Rossell J.M., et al.: 'Feasibility issues in static output-feedback controller design with application to structural vibration control', J. Frankl. Inst., 2014, 351, (1), pp. 139-155

[23] Bakka T., Karimi H.R.: ' $H_{\infty}$ static output-feedback control design with constrained information for offshore wind turbine system', J. Frankl. Inst., 2013, 350, (8), pp. 2244-2260

[24] Jafarabad A., Kashani M., Adl Parvar M.R., et al.: 'Hybrid damping systems in offshore jacket platforms with floatover deck', J. Constr. Steel Res., 2014, 98, pp. 178-187

[25] Cai M., Xiang Z., Karimi H.R.: 'Robust sampled-data $H_{\infty}$ control for vibration mitigation of offshore platforms with missing measurements', Math. Probl. Eng., 2014, Article ID 914616, pp. 1-10

[26] Kurata N., Kobori T., Takahashi M., et al.: 'Actual seismic response controlled building with semi-active damper system', Earthq. Eng. Struct. D., 1999, 28, (11), pp. 1427-1447

[27] Chopra A.K.: 'Dynamics of Structures. Theory and Applications to Earthquake Engineering' (Prentice Hall, New Jersey, 3rd ed., 2007)

[28] Balas G.J., Chiang R.Y., Packard A.K., et al.: 'MATLAB ${ }^{\mathrm{TM}}$ Robust Control Toolbox 3. User's Guide' (MathWorks Inc., Natick, Version 4.2, 2012)

[29] Kurino H., Matsunaga Y., Yamada T., et al.: High performance passive hydraulic damper with semi-active characteristics'. Proc. 13th World Conf. on Earthquake Engineering. Vancouver, Canada, 2004. Paper No. 33, pp. 1-12,

[30] Chen Y., Zhang W., Gao H.: 'Finite frequency $H_{\infty}$ control for building under earthquake excitation', Mechatronics, 2010, 20, (1), pp. 128-142

[31] Zhang H., Wang R., Wang J., et al.: 'Robust finite frequency $H_{\infty}$ static-output-feedback control with application to vibration active control of structural systems', Mechatronics, 2014, 24, (4), pp. 354-366 
[32] Hao Y., Duan Z.: 'Static output-feedback controller synthesis with restricted frequency domain specifications for time-delay systems', IET Control Theory A., 2015, 9, (10), pp. 1608-1614

[33] Du H., Lam J., Sze K.Y.: 'Non-fragile $H_{\infty}$ vibration control for uncertain structural systems', J. Sound Vib., 2004, 273, (4-5), pp. 1031-1045

[34] Zhang W., Chen Y., Gao H.: 'Energy-to-peak control for seismic-excited buildings with actuator faults and parameter uncertainties', J. Sound Vib., 2011, 330, (4), pp. 581-602

[35] Du H., Zhang N., Naghdy F.: 'Actuator saturation control of uncertain structures with input time delay', J. Sound Vib., 2011, 330, (18-19), pp. 4399-4412

[36] Shen M., Ye D., Fei S.: 'Robust $H_{\infty}$ static output control of discrete Markov jump linear systems with norm bounded uncertainties', IET Control Theory A., 2014, 8, (15), pp. 1449-1455

[37] Palacios-Quiñonero F., Rubió-Massegú J., Rossell J.M., et al.: 'Semiactive-passive structural vibration control strategy for adjacent structures under seismic excitation', J. Frankl. Inst., 2012, 349, (10), pp. 3003-3026

[38] Palacios-Quiñonero F., Rubió-Massegú J., Rossell J.M., et al.: 'Vibration control for adjacent structures using local state information', Mechatronics, 2014, 24, (4), pp. 336-344

[39] Park K.S., Ok S.Y.: 'Hybrid control approach for seismic coupling of two similar adjacent structures', J. Sound Vib., 2015, 394, pp. 1-17

[40] Zhang D., Wang X.: 'Static output feedback control of networked control systems with packet dropout', Int. J. Syst. Sci., 2012, 43, (4), pp. 665-672

[41] Zhang H., Wang J.: 'State estimation of discrete-time TakagiSugeno fuzzy systems in a network environment', IEEE T. Cyb., 2015, 45, (8), pp. 1525-1536

[42] Zhang H., Wang J.: 'Robust two-mode-dependent controller design for networked control systems with random delays modelled by Markov chains', Int. J. Control, 2015, doi:10.1080/00207179.2015.1048293, pp. 1-11 